11

\title{
Высокоселективный полосно-пропускающий фильтр на резонаторах с двухсторонним рисунком полосковых проводников на подвешенной подложке
}

\author{
(C) Б.А. Беляев ${ }^{1,2}$, А.М. Сержантов ${ }^{2}$, Я.Ф. Бальва ${ }^{1}$, Ан.А. Лексиков ${ }^{1}$, Е.О. Грушевский ${ }^{1}$ \\ ${ }^{1}$ Институт физики им. Л.В. Киренского СО РАН, Красноярск, Россия \\ ${ }^{2}$ Сибирский федеральный университет, Красноярск, Россия \\ E-mail: belyaev@iph.krasn.ru
}

Поступило в Редакцию 15 января 2019г.

В окончательной редакции 15 января 2019 г.

Принято к публикации 20 февраля 2019г.

Исследована новая конструкция резонатора, образованного шпильковым полосковым проводником со шлейфом, расположенным на одной поверхности диэлектрической подложки, и регулярными замкнутыми на экран с одного конца полосковыми проводниками на другой поверхности. В резонаторе собственные частоты первых трех мод колебаний можно сблизить, при этом резонансы двух мод участвуют в формировании полосы пропускания, а резонанс третьей моды формирует минимум коэффициента передачи рядом с полосой пропускания. Конструкция, изготовленная из четырех таких резонаторов, обладает характеристикой фильтра восьмого порядка и при внутренних размерах корпуса $45 \times 16 \times 6.25 \mathrm{~mm}$ имеет центральную частоту полосы пропускания $f_{0}=0.52 \mathrm{GHz}$ при ее относительной ширине $14 \%$. Фильтр обладает высокой селективностью благодаря близкому расположению к полосе пропускания полюсов затухания и широкой высокочастотной полосой заграждения, простирающейся до частоты более чем $5 f_{0}$ по уровню $-100 \mathrm{~dB}$.

DOI: 10.21883/PJTF.2019.10.47749.17703

Хорошо известно, что полосно-пропускающие фильтры сверхвысоких частот (СВЧ) относятся к важнейшим элементам систем связи, радиолокации, радионавигации и специальной радиоаппаратуры $[1,2]$. При этом фильтры нередко определяют габариты, а главное, качество радиотехнических СВЧ-устройств, напрямую зависящее от частотно-селективных характеристик фильтров. Требования к характеристикам фильтров, в первую очередь к крутизне склонов полосы пропускания, величине затухания в полосах заграждения, а также к ширине высокочастотной полосы заграждения, непрерывно повышаются, поэтому разработка новых миниатюрных конструкций фильтров с высокой избирательностью представляет собой важную и актуальную задачу.

Известно также, что конструкции фильтров на основе микрополосковых или полосковых резонаторов на подвешенной подложке являются одними из самых миниатюрных [1-3]. Как правило, в любой конструкции для повышения крутизны склонов полосы пропускания амплитудно-частотной характеристики (АЧХ) разработчики увеличивают порядок фильтра, наращивая количество резонаторов в нем [4], добавляют шлейфные элементы [5] или используют многомодовые резонаторы [6], также позволяющие увеличить порядок фильтра без увеличения числа резонаторов. При этом одновременно повышается уровень затухания в полосах заграждения фильтра. Однако для увеличения ширины высокочастотной полосы заграждения, которая ограничивается резонансами высших мод колебаний, формирующих паразитные полосы пропускания, часто используются нерегулярные резонаторы [7-9], резонаторы на квазисосре- доточенных элементах, изготовленных на подвешенной подложке [10] или по LTCC-технологии $[11,12]$.

Один из наиболее эффективных подходов, позволяющий одновременно значительно расширить высокочастотную полосу заграждения, увеличить затухание в ней и уменьшить размеры планарных полоснопропускающих фильтров, заключается в использовании многопроводниковых полосковых резонаторов $[13,14]$. Однако на АЧХ таких конструкций отсутствуют полюса затухания, увеличивающие крутизну склонов полосы пропускания, поэтому повысить избирательность фильтров на таких конструкциях можно только наращиванием числа звеньев. Кроме того, процесс изготовления фильтров на основе многопроводниковых резонаторов - сложная задача, требующая специального технологичного оборудования высокого уровня.

В настоящей работе исследован фильтр, в котором используется новая конструкция миниатюрного полоскового резонатора на подвешенной подложке с двухсторонним рисунком полосковых проводников (рис. 1,a), размещенного в металлическом экране 5. Резонатор образован шпильковым полосковым проводником 2 на одной стороне подложки 1 , к центру которого подключен шлейф 3. На второй стороне подложки расположены полосковые проводники 4, замкнутые одним концом на экран. Эти проводники расположены строго напротив разомкнутых концов полосковой структуры на первой стороне подложки, имеют соответствующую им ширину и вместе с ними образуют квазисосредоточенные емкости, существенно уменьшающие размеры резонатора. 
$a$
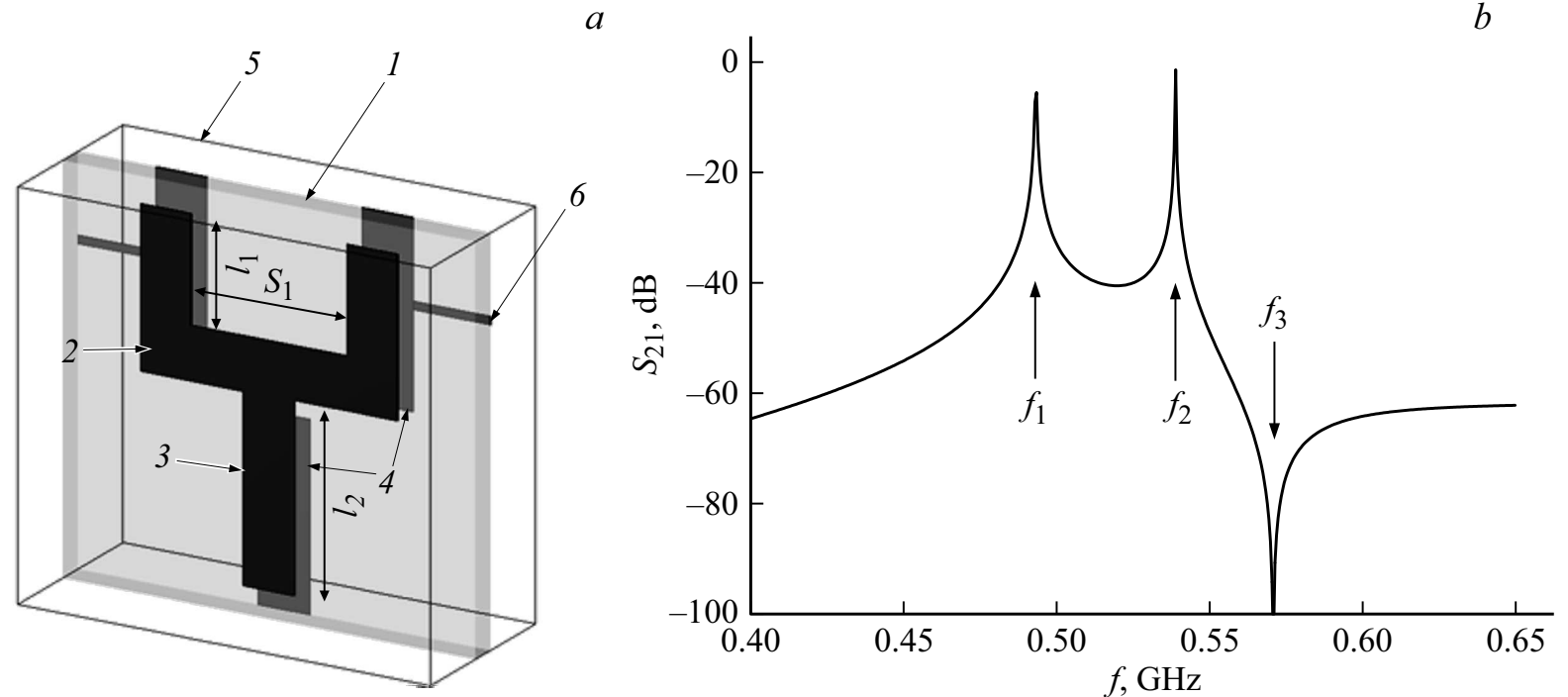

Рис. 1. $a-$ конструкция полоскового резонатора. 1 - подвешенная диэлектрическая подложка, $2-$ шпильковый полосковый проводник, 3 - шлейф, 4 - регулярные полосковые проводники, 5 - металлический корпус, 6 - линии передачи для подключения портов входа и выхода. $b-$ АЧХ резонатора при слабой связи с портами.

Порты входа и выхода с волновым сопротивлением $50 \Omega$ подключены к полосковым линиям 6 .

На рис. 1, $b$ приведена АЧХ исследуемого резонатора, рассчитанная с помощью электродинамического анализа 3D-модели конструкции в пакете программ CST-microwave Studio для случая ее слабой связи с портами. Резонатор имел следующие конструктивные параметры: относительная диэлектрическая проницаемость подложки из высокочастотной керамики ТБНС $\varepsilon=80$, толщина подложки $0.25 \mathrm{~mm}$, расстояние от экранов до поверхности подложки $3 \mathrm{~mm}$, ширина полосковых проводников $1 \mathrm{~mm}$, размеры структуры $l_{1}=5.9 \mathrm{~mm}$, $l_{2}=7.0 \mathrm{~mm}, S_{1}=6.0 \mathrm{~mm}$. При этом отступы свободных концов полосковых проводников „шшпильки“ и шлейфа от краев подложки были по $0.5 \mathrm{~mm}$. Как показали исследования, рассматриваемый резонатор является трехмодовым с близкими собственными частотами $f_{1}, f_{2}$ и $f_{3}$. При этом отметим, что резонансы двух мод колебаний на частотах $f_{1}$ и $f_{2}$ участвуют в формировании полосы пропускания фильтра, построенного на таких резонатоpax, а резонанс третьей моды на частоте $f_{3}$ формирует полюс затухания рядом с полосой пропускания. Важно заметить, что при выборе длины шлейфа резонатора частоту $f_{3}$ можно изменять, располагая полюс затухания либо слева, либо справа от полосы пропускания, увеличивая тем самым крутизну соответствующего склона АЧХ.

На рис. 2 показана топология проводников конструкции фильтра на подвешенной подложке, имеющей толщину $0.25 \mathrm{~mm}$ и $\varepsilon=80$, построенного на четырех резонаторах с зазорами между полосковыми проводниками наружных пар резонаторов $S_{e}$ и зазором между проводниками внутренней пары резонаторов $S_{i}$. Верхний и нижний экраны располагаются на расстоянии $3 \mathrm{~mm}$ до поверхностей подложки. Следует отметить, что в рассматриваемой четырехзвенной конструкции фильтра на шпильковых резонаторах со шлейфами в области первой полосы пропускания существует двенадцать мод колебаний с близкими собственными частотами (по три моды от каждого резонатора). При этом резонансы восьми мод колебаний формируют полосу пропускания, а резонансы остальных четырех мод формируют полюса затухания слева и справа вблизи полосы пропускания. Поэтому у такого четырехзвенного фильтра крутизна склонов АЧХ значительно выше, чем у фильтра на восьми шпильковых резонаторах без шлейфных элементов.

Настройка фильтра осуществлялась „ручным“ параметрическим синтезом с использованием численного электродинамического анализа его 3D-модели в пакете программ CST-microwave Studio. При этом центральная частота полосы пропускания фильтра настраивалась размерами шпильковых проводников резонаторов и длиной соответствующих им полосковых проводников на обратной стороне подложки, замкнутых одним концом на экран. Ширина полосы пропускания настраивалась зазорами между резонаторами $S_{e}$ и $S_{i}$. Точки кондуктивного подключения отрезков линий, соединяющих крайние резонаторы с портами, имели смещение относительно конца наружного проводника „шпильки“ на величину $l_{c}$ (рис. 2), что позволяло настраивать заданный уровень потерь на отражение СВЧ-мощности в полосе пропускания. Длиной шлейфов и длиной соответствующих им полосковых проводников на обратной стороне подложки настраивались частоты полюсов затухания так, чтобы они располагались справа и слева вблизи полосы пропускания, обеспечивая высокую крутизну склонов и близкую к симметричной форму АЧХ. 


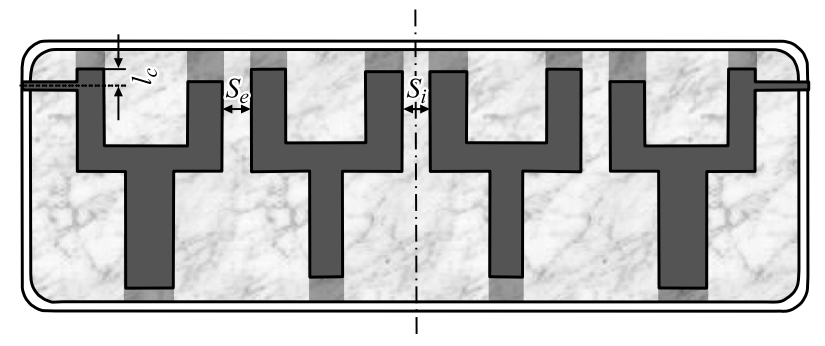

Pис. 2. Топология полосковых проводников фильтра на четырех резонаторах.
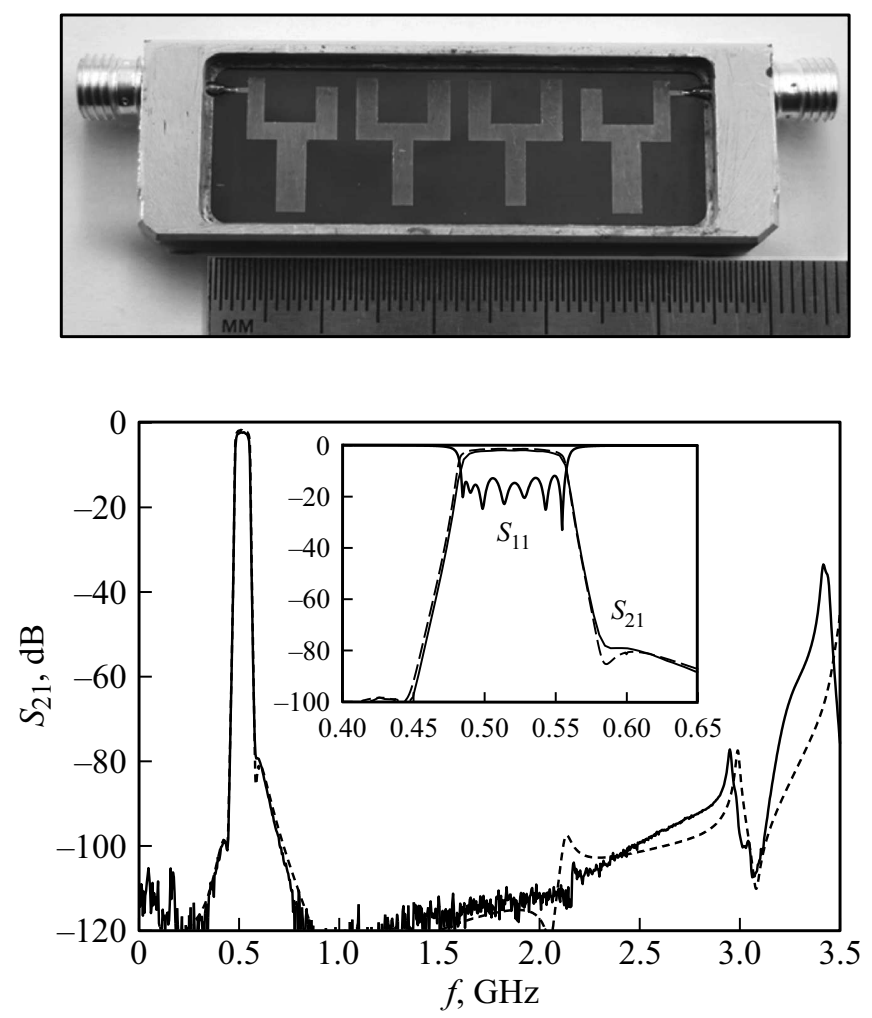

Рис. 3. Измеренные (сплошные линии) и рассчитанные (штриховые линии) частотные зависимости потерь на отражение $S_{11}$ и на прохождение $S_{21}$ действующего макета фильтра. Сверху представлена фотография устройства без верхней крышки.

Для определенности был синтезирован фильтр канала связи с центральной частотой полосы пропускания $f_{0}=0.52 \mathrm{GHz}$ при ее относительной ширине, измеренной по уровню $3 \mathrm{~dB}$ от уровня минимальных потерь, $\Delta f / f_{0}=14 \%$, уровень отраженной от входа СВЧ-мощности не превышал $-14 \mathrm{~dB}$. Амплитудночастотная характеристика синтезированного фильтра представлена штриховой линией на рис. 3. Сплошной линией на этом рисунке показана АЧХ экспериментального образца фильтра, изготовленного в соответствии с конструктивными параметрами, полученными в результате синтеза. Размеры диэлектрической подложки филь- тра составляли $16 \times 45 \mathrm{~mm}$. Сверху на рис. 3 показана фотография фильтра без верхней крышки-экрана.

Видно, что представленные зависимости демонстрируют довольно хорошее согласие теории и эксперимента. При этом центральная частота полосы пропускания экспериментального образца практически совпадает с расчетной, а относительная ширина полосы пропускания оказалась немного меньше: примерно на $0.5 \%$. Отметим также, что измеренные минимальные потери для экспериментального образца в полосе пропускания фильтра всего на $0.4 \mathrm{~dB}$ превышают расчетные и составляют $\sim 1.9 \mathrm{~dB}$.

Таким образом, разработанная конструкция фильтра демонстрирует не только широкую высокочастотную полосу заграждения, простирающуюся по уровню $-100 \mathrm{~dB}$ до частоты более чем $5 f_{0}$, но и высокую крутизну склонов АЧХ. В представленном на рис. 3 фильтре крутизна склонов АЧХ примерно одинаковая, что доказывают коэффициенты крутизны низкочастотного $K_{l}=4.1$ и высокочастотного $K_{h}=4.0$ склонов, которые вычислялись по формулам [4]:

$$
K_{l}=\frac{\Delta f_{3} / 2}{\Delta f_{l}-\Delta f_{3} / 2}, \quad K_{h}=\frac{\Delta f_{3} / 2}{\Delta f_{h}-\Delta f_{3} / 2} .
$$

Здесь $\Delta f_{3}$ - ширина полосы пропускания фильтра по уровню $-3 \mathrm{~dB}$, а $\Delta f_{l}$ и $\Delta f_{h}-$ ширины полос частот, измеренные от центра полосы пропускания $f_{0}$ соответственно до частоты низкочастотного $f_{l}$ и высокочастотного $f_{h}$ склонов АЧХ на уровне $-30 \mathrm{~dB}$ от уровня минимальных потерь. Очевидно, что равенство коэффициентов крутизны $K_{l}$ и $K_{h}$ свидетельствует о симметричной форме амплитудно-частотной характеристики синтезированного фильтра. Однако важно отметить еще одно достоинство разработанной конструкции: она позволяет создавать фильтры с асимметричной формой АЧХ путем значительного увеличения крутизны одного из склонов. Для этого достаточно всего лишь подбором длины шлейфов все полюса затухания разместить вблизи соответствующего склона АЧХ.

Заметим, что протяженность высокочастотной полосы заграждения разработанной конструкции фильтра увеличивается с уменьшением толщины подложки. При этом одновременно уменьшается длина резонаторов, а соответственно и габариты всего фильтра. В меньшей степени протяженность высокочастотной полосы заграждения растет с увеличением ширины полосковых проводников и увеличением расстояния от поверхностей подвешенной подложки до экранов.

\section{Финансирование работы}

Работа выполнена при поддержке Министерства образования и науки РФ (соглашение № 14.575.21.0142, уникальный идентификатор проекта RFMEFI57517X0142). 


\section{Список литературы}

[1] Hunter I.C. Theory and design of microwave filters. IET Electromagnetic Waves Ser. V. 48. Cambridge: University Press, 2006. $353 \mathrm{p}$.

[2] Morgan M.A. Reflectionless filters. Boston-London: Artech House Microwave Library, 2017. $258 \mathrm{p}$.

[3] Hong $J-S$. Microstrip filters for $\mathrm{RF} /$ microwave applications. Hoboken: John Wiley \& Sons, Inc., 2011. 635 p.

[4] Беляев Б.А., Лексиков А.А., Тюрнев В.В. // Радиотехника и электроника. 2004. Т. 49. № 11. С. 1315-1324.

[5] Александровский А.А., Беляев Б.А., Лексиков А.А. // Радиотехника и электроника. 2003. Т. 48. № 4. С. 398-405.

[6] Беляев Б.А., Ходенков С.А., Лексиков Ан.А., Шабанов В.Ф. // ДАН. 2017. Т. 474. № 6. С. 682-686.

[7] Wei X.B., Shi Y., Wang P., Liao J.X., Xu Z.Q., Yang B.C. // J. Electromagn. Waves Appl. 2012. V. 26. P. 1095-1104.

[8] Kuo J.-T., Shih E. // IEEE Trans. Microwave Theory Techn. 2003. V. 51. N 5. P. $1554-1559$.

[9] Lin S.-C., Deng P.-H., Lin Y.-S., Wang C.-H., Chen C.H. // IEEE Trans. Microwave Theory Techn. 2006. V. 54. N 3. P. 1011-1017.

[10] Беляев Б.А., Волошин А.С., Булавчук А.С., Галеев Р.Г. // Письма в ЖТФ. 2016. Т. 42. В. 12. С. 30-37.

[11] Холодняк Д.В., Тургалиев В.М., Мунина И.В., Туральчук П.А., Вендик И.Б. // Радиотехника. 2012. № 7. C. $132-137$.

[12] Симин А., Холодняк Д., Вендик И. // Компоненты и технологии. 2005. № 5. С. 190-196.

[13] Belyaev B.A., Serzhantov A.M., Tyurnev V.V., Bal'va Y.F., Leksikov A.A. // Prog. Electromagn. Res. C. 2014. V. 48. P. 37-44.

[14] Belyaev B.A., Serzhantov A.M., Leksikov A.A., Balva Y.F., Leksikov A.A. // Microwave Opt. Technol. Lett. 2017. V. 59. P. $2212-2216$. 